\title{
Risk assessment of pharmaceutically active compounds (PhACs) in the Klang River estuary, Malaysia
}

\begin{abstract}
ABSTRCT
The concentration profile, distribution and risk assessment of pharmaceutically active compounds (PhACs) in the coastal surface water from the Klang River estuary were measured. Surface coastal water samples were extracted using offline solid phase, applying polymeric C18 cartridges as extraction sorbent and measuring with liquid chromatography mass spectrometrymass spectrometry (LC MS-MS) technique. Extraction method was optimized for its recovery, sensitivity and linearity. Excellent recoveries were obtained from the optimized method with percentage of recoveries ranging from 73 to $126 \%$. The optimized analytical method achieved good sensitivity with limit of detection ranging from 0.05 to $0.15 \mathrm{ng} \mathrm{L}-1$, while linearity of targeted compounds in the LC MS-MS system was more than 0.990 . The results showed that amoxicillin has the highest concentration (102.31 ng L-1) followed by diclofenac (10.80 ng L-1) and primidone (7.74 ng L-1). The percentage of contribution (\% of total concentration) for the targeted $\mathrm{PhACs}$ is in the following order; amoxicillin $(92.90 \%)>$ diclofenac $(3.95 \%)>$ primidone $(1.23 \%)>$ dexamethasone $(0.75 \%)>$ testosterone $(0.70 \%)>$ sulfamethoxazole $(0.33 \%)>$ progesterone $(0.14 \%)$. Environmental risk assessment calculated based on deterministic approach (the RQ method), showed no present risk from the presence of PhACs in the coastal water of Klang River estuary. Nonetheless, this baseline assessment can be used for better understanding on PhACs pollution profile and distribution in the tropical coastal and estuarine ecosystem as well as for future comparative studies.
\end{abstract}

Keyword : Coastal water; Emerging organic contaminants; Klang River estuary; Pharmaceutically active compounds (PhACs); Risk assessment. 